\title{
DEGRADAĈ̃̃O DE $\alpha$-TOCOFEROL, RETINOL E ÁCIDO ASCÓRBICO DURANTE PROCESSAMENTO E ARMAZENAMENTO DE RAÇÕES PARA CAMARÃO
}

\author{
$\alpha$-Tocopherol, retinol and ascorbic acid degradation during \\ the processing and storage of shrimp food
}

\author{
Zilmara Vieira Pedrosa ${ }^{1}$, José Marcelino Oliveira Cavalheiro' ${ }^{2}$, \\ João Paulo de Sousa Prado ${ }^{2}$, Valdir Mendes Soares de Oliveira ${ }^{2}$
}

\begin{abstract}
RESUMO
O cultivo de camarão marinho é um setor de grande viabilidade econômica, principalmente na região Nordeste. Entretanto, a alimentação dos camarões é responsável pelo maior custo no cultivo desses crustáceos, a ração deve ser nutricionalmente eficiente, sendo indispensável a presença das vitaminas que são substâncias fundamentais para o funcionamento do organismo animal, e sua falta resulta frequentemente em crescimento e desenvolvimento deficiente. $\mathrm{O}$ retinol, $\alpha$-tocoferol e o ácido ascórbico são vitaminas que atuam em diversos processos metabólicos. São indispensáveis em rações animais porque são antioxidantes que auxiliam os animais contra o estresse oxidativo. Objetivou-se, neste trabalho, avaliar as perdas das vitaminas durante o processamento das dietas e no período de armazenagem. Foram formuladas oito dietas isoprotéicas com diferentes níveis de vitamina $\mathrm{A}, \mathrm{E}$ e $\mathrm{C}$ que foram processadas com temperatura de até $80^{\circ} \mathrm{C}$. Após a fabricação e depois de 60 dias de armazenagem foram analisadas e quantificadas as vitaminas a fim de descobrir as perdas durante o processamento e após estocagem. Constatou-se que a vitamina A, que foi adicionada na dieta, foi perdida em até $99,56 \%$. Já a vitamina E perdeu no máximo 93,5\% e a vitamina C com 70\% de perda, durante o processamento. Durante o período de armazenagem as perdas foram menores. Pode-se concluir que as vitaminas são extremamente sensíveis aos fatores que atuam durante a manufatura das dietas, ocasionando perdas consideráveis de vitaminas.
\end{abstract}

Termos para indexação: Alimentação animal, vitaminas, estabilidade.

\begin{abstract}
The farming of marine shrimp is a sector of great economic viability, mainly in the Northeast region of Brazil. However, feeding is responsible for the major cost in the farming of these crustaceans, so the food must be nutritiously efficient, since it is essential to the presence of vitamins which are vital substances to the functioning of the animal's organism where its lack frequently results in deficient growth and development. Retinol, $\alpha$-tocopherol, and ascorbic acid are vitamins that act in several metabolic processes, therefore they are indispensable in animal food due to their antioxidant effects that protect these animals against oxidative stress. The objective of this study was to evaluate the vitamin losses during the diet processing and in the storage period. Eight isoproteic diets were formulated with different levels of vitamin $\mathrm{A}, \mathrm{E}$ and $\mathrm{C}$ that were processed with temperatures up to $70^{\circ} \mathrm{C}$. After the production and 60 days of storage, the vitamins were analyzed and quantified in order to discover the losses during the processing and post-storage. It was observed that the vitamin A suffered loss of up to $99.56 \%$, vitamin E had its maximum loss value of $93.5 \%$ and vitamin $\mathrm{C}$ with $70 \%$ of loss, during the processing. During the storage period the losses were lower. It can be concluded that the vitamins are extremely sensitive to the factors that occur during the production and food storage, causing considerable vitamin losses.
\end{abstract}

Index terms: Animal feeding, vitamins, stability.

(Recebido em 21 de setembro de 2009 e aprovado em 10 de agosto de 2010)

\section{INTRODUÇÃO}

O cultivo de camarões ou carcinicultura vem ganhando destaque nos últimos anos como uma alternativa economicamente importante para o Brasil, principalmente nos estados do Nordeste. O litoral nordestino é considerado ideal para o cultivo de camarões marinhos devido à extensa área costeira com temperaturas elevadas, o que possibilita a criação de camarões durante todo o ano (Nunes, 2001; Barbieri Júnior \& Ostrensky Neto, 2002).
O avanço da carcinicultura está intimamente relacionado com a disponibilidade de rações balanceadas. Os camarões necessitam de nutrientes ditos essenciais que precisam ser adicionados à dieta. Dentre esses nutrientes temos as vitaminas que são compostos que auxiliam no crescimento, na reprodução e na manutenção da vida dos animais (Boyd, 2001; Waldige, 2003; Ribeiro \& Seravalli, 2004).

As dietas de camarões frequentemente são preparadas com grandes quantidades de vitaminas, existem

\footnotetext{
1Universidade Federal da Paraíba/UFPB - 58059-900 - Jardim Cidade Universitária - João Pessoa, PB - zilfarma@yahoo.com.br

2Universidade Federal da Paraíba/UFPB - João Pessoa, PB
} 
diferentes razões para isso, entre elas podemos citar: informações insuficientes sobre os requerimentos de vitaminas para camarões; os camarões se alimentam lentamente e o alimento permanece na água durante várias horas onde as vitaminas hidrossolúveis se solubilizam; algumas vitaminas são destruídas durante a preparação e o armazenamento da dieta (Conklin, 1997; Akiyama et al., 1992).

Esses micronutrientes são facilmente oxidados em condições neutras ou alcalinas em certas condições como a presença de oxigênio, umidade, microelementos, temperaturas elevadas, luz e lipídios oxidados (Leite et al., 2008).

A vitamina A é usada nas formas de ésteres como acetato, propionato ou palmitato nas rações dos animais. Ela auxilia na formação do sistema da visão, no crescimento, desenvolvimento ósseo, manutenção do tecido epitelial e no sistema de imunidade, logo aumenta a sobrevivência dos animais (Bispo \& Pereira, 1994; Faria \& Junqueira, 2000).

Peil et al. (2007), estudando os efeitos da adição de diferentes níveis de vitamina A na alimentação de póslarvas de jundiá, observaram que o incremento vitamínico foi bastante significativo para o aumento da sobrevivência dos animais, e obteve o melhor desempenho em sobrevivência, o nível de maior adição de vitamina A.

A vitamina $\mathrm{E}$ tem como principal representante o $\alpha$-tocoferol, sendo usada para fortificação da alimentação de peixes e camarões, com o objetivando-se melhorar o crescimento, a resistência ao estresse e a doenças, garantido a sobrevivência desses animais (Huo et al., 1999).

A principal atividade dos tocoferóis é sua ação antioxidante, sendo muito importante na prevenção da oxidação da vitamina A e de ácidos graxos insaturados (Sackheim \& Lehman, 2001).

Com um estudo objetivou-se que camarões da espécie Penaeus vannamei apresentou grande perda de peso e alta mortalidade quando alimentados com dietas pobres em vitamina E, comparando ao tratamento suplementado com $1600 \mathrm{mg}$ de vitamina $\mathrm{E} / \mathrm{kg}$ de ração, indicando que a vitamina $\mathrm{E}$ é indispensável para o crescimento adequado e a sobrevivência (He, 1992).

O ácido ascórbico faz parte do grupo dos antioxidantes e muitos estudos demonstram que o ácido ascórbico estimula o sistema imunológico de animais aquáticos. Um fator que dificulta a preservação desta vitamina na ração é a sua instabilidade quando exposta a algumas condições como luminosidade, oxigênio, temperatura e água. A exposição a estes fatores acarreta a perda da mesma (Lee \& Shiau, 2002; Guilherme et al., 2009).

O objetivou-se, no presente estudo, do presente estudo foi avaliar as perdas das vitaminas A, E e C existentes nas rações utilizadas para nutrição de camarões, durante o processo de manufatura e armazenagem das dietas.

\section{MATERIAIS E MÉTODOS}

Formularam-se oito dietas isoprotéicas com diferentes níveis de vitamina A, E e C (Tabela 1). A elaboração da ração foi baseada no método descrito por Gadelha et al. (2010), em que todos os ingredientes utilizados, exceto o premix de Retinol, $\alpha$-tocoferol, ácido ascórbico e óleo de peixe foram misturados em batedeira convencional. Em seguida foi adicionado o premix de ácido ascórbico diluído em água, o premix de Retinol diluído em óleo de peixe. O premix de á-tocoferol foi misturado com os outros ingredientes. A massa foi umedecida lentamente com água à $70^{\circ} \mathrm{C}$ para aglutinar a ração. Essa massa foi introduzida em peletilizador, onde foram obtidos os peletes com $1 \mathrm{~mm}$ de diâmetro.

As rações elaboradas foram analisadas em triplicata, logo após o processamento e depois de um período de 60 dias de armazenagem à temperatura

Tabela 1 - Níveis de vitaminas adicionadas nas rações elaboradas.

\begin{tabular}{cccc}
\hline Ração & Retinol (UI/Kg) & $\alpha$-Tocoferol $(\mathrm{UI} / \mathrm{Kg})$ & ácido ascórbico $\mathrm{mg} / \mathrm{Kg}$ \\
\hline $\mathrm{R}_{1}$ & 0 & 184 & 0 \\
$\mathrm{R}_{2}$ & 0 & 0 & 240 \\
$\mathrm{R}_{3}$ & 0 & 0 & 480 \\
$\mathrm{R}_{4}$ & 0 & 364 & 0 \\
$\mathrm{R}_{5}$ & 10000 & 0 & 0 \\
$\mathrm{R}_{6}$ & 10000 & 184 & 240 \\
$\mathrm{R}_{7}$ & 20000 & 0 & 0 \\
$\mathrm{R}_{8}$ & 20000 & 364 & 480 \\
\hline
\end{tabular}


ambiente a fim de determinar as perdas vitamínicas. As vitaminas A e E foram quantificadas por HPLC e o ácido ascórbico foi determinado pelo método titulométrico descrito por Rangana (1979).

Para as análises de vitamina A e E, as amostras de ração foram previamente trituradas, e logo depois extraídas com hexano seguindo a metodologia de Gomis et al. (2000). Para a extração das formas vitamínicas das matrizes utilizouse etanol com 0,1\% de Butil Hidroxi Anisol (BHA), seguida de partição com hexano. A fração do hexano foi secada em nitrogênio e ressuspendida em metanol. Em seguida, foram filtrados em membranas Millipore Fluoropore de $0,5 \mu \mathrm{m}$ de poro, para serem, depois, injetados no cromatógrafo. Para separação das vitaminas foi utilizada coluna de fase reversa e um sistema isocrático. Para a análise cromatográfica foi utilizado o método de Qian \& Sheng (1998) para vitamina E, e para vitamina A foi baseado no método descrito pela United States Pharmacopeia (2004). Como fase móvel, foi utilizada para vitamina E uma proporção de 28:68:4 (acetonitrila:metanol:água), a um fluxo de 1,7 mL/min, com detecção em $208 \mathrm{~nm}$. Para vitamina A, foi utilizada uma proporção de 98:2 (metanol:água), a um fluxo de $1 \mathrm{~mL} / \mathrm{min}$, com detecção em $325 \mathrm{~nm}$. Para identificação, utilizou-se a comparação dos tempos de retenção obtidos com os padrões nas mesmas condições cromatográficas e os espectros de absorção obtidos no detector de arranjo de diodos (DAD). A quantificação foi realizada pelo método de padronização externa.

O equipamento de cromatografia líquida utilizado foi um Varian 2699, composto por bomba isocrática, com injetor manual Reody, com alça de amostragem de $2 \mu \mathrm{l}$, acoplado a detector de arranjo de diodo (DAD). A coluna cromatográfica utilizada foi: $\mathrm{C}_{18}$ (Chrompack-Varian, Inerstisil - 150 x 4,6 mm 5ODS-2), com $5 \mu \mathrm{m}$ de diâmetro de partícula.

Os resultados foram tratados estatisticamente mediante a análise de variância (ANOVA) e aplicado o teste de Duncan entre as médias, a 5\% de probabilidade, por meio do programa estatístico SPSS, versão 14.0 (SPSS, 2001).

\section{RESULTADOS E DISCUSSÃO}

Constatou-se que as rações que receberam algum nível de vitamina apresentaram perdas significativas, principalmente durante o processamento da dieta.

As rações que foram adicionadas $\alpha$-Tocoferol apresentaram perdas tanto durante o processamento quanto no período de estocagem. As dietas R1e R6 que receberam 184UI/Kg dessa vitamina, perderam 90,4 e 90,5\%, respectivamente, durante o processo de manufatura. Já durante a armazenagem as perdas foram menores, 31,2 e $39,6 \%$. Os valores de vitamina $\mathrm{E}$ quantificados nas rações R1 e R6 não apresentaram diferenças estatísticas, ao nível de $5 \%$ de probabilidade pelo teste de Duncan.

As rações R4 e R8 em que foram adicionados 364UI/Kg de vitamina $\mathrm{E}$, detectamos perdas que variaram de 93,5 a $90,6 \%$, respectivamente, quando analisadas após o processamento. O período de armazenagem dessas dietas proporcionou perdas de 20,4 e $29 \%$ desta vitamina, havendo diferença significativa entre essas dietas, ao nível de 5\% de significância, pelo teste de Duncan (Tabela 2).

Prado (2008), estudando a estabilidade de vitamina A e E em rações comerciais para camarão, observou que o conteúdo de vitamina E quantificado era muito inferior ao que estava informado no rótulo do produto. As três rações analisadas apresentaram perdas superiores a $96 \%$, sendo essas perdas ocasionadas provavelmente durante a manufatura dos produtos, bem como no período de estocagem. Observou-se, ainda, que à temperatura ambiente $\left(20 \pm 2^{\circ} \mathrm{C}\right)$, uma das rações apresentou perda de $25 \%$ dessa vitamina num período de 30 dias, enquanto as outras tiveram perdas de até $88 \%$, justificando a variação com o nível de proteção física ocasionada pela estrutura da ração (peletes).

Tabela 2 - Conteúdo de $\alpha$-tocoferol adicionado nas rações e teores obtidos após o processamento e depois do período de manejo e estocagem.

\begin{tabular}{cccccc}
\hline \multicolumn{5}{c}{ Conteúdo de $\alpha$-Tocoferol nas formulações (UI/kg) } \\
\hline Rações & Antes processamento & Após processamento & Perdas $(\%)$ & Após estocagem (60dias) & Perdas $(\%)$ \\
\hline R1 & 184.0 & $17.71^{\mathrm{c}} \pm 0.95$ & 90.4 & $12.19^{\mathrm{c}} \pm 1.79$ & 31.2 \\
R4 & 364.0 & $23.51^{\mathrm{b}} \pm 2.68$ & 93.5 & $18.72^{\mathrm{b}} \pm 3.91$ & 20.4 \\
R6 & 184.0 & $17.43^{\mathrm{c}} \pm 0.63$ & 90.5 & $10.53^{\mathrm{c}} \pm 2.07$ & 39.6 \\
R8 & 364.0 & $34.30^{\mathrm{a}} \pm 3.57$ & 90.6 & $24.39^{\mathrm{a}} \pm 2.20$ & 29.0 \\
\hline
\end{tabular}

*Letras diferentes na mesma coluna diferem significativamente, ao nível de 5\% pelo teste de Duncan. 
Garcia et al. (2005) analisaram a estabilidade de vitamina $\mathrm{A}, \mathrm{E}$ e $\mathrm{C}$ adicionadas à balas de gelatina e observaram que ocorreram perdas de 25,12 e $1 \%$ após o processo de manufatura desse produto, em que utilizaram temperaturas de $70^{\circ} \mathrm{C}$. Mostrando-se diferente dos resultados obtidos neste estudo reforçando portanto, a idéia de que matrizes diferentes interferem nessas perdas.

Pelos resultados obtidos das análises do retinol (Tabela 3) observaram-se perdas dessa vitamina superiores a $99 \%$, durante a manufatura. As dietas R5 e R6 que receberam $10000 \mathrm{UI} / \mathrm{Kg}$ de retinol apresentaram degradação de 99,54 e 99,22\% no processamento e para as dietas R7 e $\mathrm{R} 8$ os valores encontrados foram de 99,22 e $99,40 \%$. Durante a estocagem das dietas as perdas de vitamina das dietas R5 e R6 foram de 52,72 e 75,51\%. Já as dietas R7 e R8 apresentaram perdas de 81,11 e 82,08\%.

Prado (2008), observando a estabilidade da vitamina A presente na ração no ambiente de estufa à uma temperatura de $40 \pm 2^{\circ} \mathrm{C}$, obteve degradação de até $77 \%$ da vitamina, portanto é possível uma perda de mais de $99 \%$, quando a amostra é submetida ao processamento que utiliza temperatura de até $70^{\circ} \mathrm{C}$.
Com as análises das dietas quanto à presença de ácido ascórbico observa-se que as rações R2 e R6 que receberam $240 \mathrm{mg} / \mathrm{Kg}$ de vitamina C, perderam 48,2 e 66,2\% dessa vitamina durante a fabricação das dietas. Já durante a estocagem, as perdas foram de 72,7 e $68 \%$. As rações R3 e R8 apresentaram perdas de 66,2 e $70 \%$, durante o processamento. No período de estocagem, os resultados foram de 62,8 e 64,8\%, conforme Tabela 4.

Marchetti et al. (1999) estudando a estabilidade de vitaminas na forma cristalina e revestidas em rações para peixe, observaram uma perda de ácido ascórbico na forma cristalina de $48,3 \%$ quando as rações foram expostas à peletização. Logo, podemos observar que a menor perda de vitamina $\mathrm{C}$ observada após o processo de peletização nesta pesquisa foi de $48,2 \%$, estando de acordo com o obtido no trabalho dos demais autores. As perdas superiores que foram encontradas podem ser justificadas pela temperatura utilizada durante o processamento, enquanto esse trabalho foi usada uma temperatura de até $70^{\circ} \mathrm{C}$, e na pesquisa citada anteriormente foi utilizada uma temperatura máxima de $68^{\circ} \mathrm{C}$.

Tabela 3 - Conteúdo de Retinol adicionado às rações e teores obtidos após o processamento e depois do período de manejo e estocagem.

\begin{tabular}{cccccc}
\hline \multicolumn{5}{c}{ Conteúdo de Retinol nas formulações (UI/kg) } \\
\hline Rações & Antes processamento & Após processamento & Perdas $(\%)$ & Após estocagem (60dias) & Perdas $(\%)$ \\
\hline R5 & 10000 & $45.62^{\mathrm{d}} \pm 0.78$ & 99,54 & $21.57^{\mathrm{b}} \pm 0.51$ & 52,72 \\
R6 & 10000 & $78.32^{\mathrm{c}} \pm 1.90$ & 99,22 & $19.18^{\mathrm{b}} \pm 0.37$ & 75,51 \\
R7 & 20000 & $156.67^{\mathrm{a}} \pm 0.09$ & 99,22 & $29.59^{\mathrm{a}} \pm 0.98$ & 81,11 \\
R8 & 20000 & $120.44^{\mathrm{b}} \pm 4.14$ & 99,40 & $21.58^{\mathrm{b}} \pm 0.55$ & 82,08 \\
\hline
\end{tabular}

*Letras diferentes na mesma coluna diferem significativamente, ao nível de 5\% pelo teste de Duncan.

Tabela 4 - Conteúdo de ácido ascórbico adicionado às rações e teores obtidos após o processamento e depois do período de manejo e estocagem.

\begin{tabular}{cccccc}
\hline \multicolumn{5}{c}{ Conteúdo de ácido ascórbico nas formulações $(\mathrm{mg} / \mathrm{kg})$} \\
\hline Rações & Antes processamento & Após processamento & Perdas $(\%)$ & Após estocagem (60dias) & Perdas (\%) \\
\hline R2 & 240 & $124.28^{\mathrm{c}} \pm 1.55$ & 48,2 & $33.95^{\mathrm{c}} \pm 0.60$ & 72,7 \\
R3 & 480 & $162.38^{\mathrm{a}} \pm 0.99$ & 66,2 & $60.38^{\mathrm{a}} \pm 1.84$ & 62,8 \\
R6 & 240 & $81.18^{\mathrm{d}} \pm 2.54$ & 66,2 & $25.98^{\mathrm{d}} \pm 0.39$ & 68,0 \\
R8 & 480 & $144.25^{\mathrm{b}} \pm 5.41$ & 70,0 & $50.79^{\mathrm{b}} \pm 0.57$ & 64,8 \\
\hline
\end{tabular}

*Letras diferentes na mesma coluna diferem significativamente, ao nível de 5\% pelo teste de Duncan. 


\section{CONCLUSÕES}

As vitaminas adicionadas às formulações apresentaram alta taxa de degradação durante o processamento e o período de estocagem das dietas, sendo as maiores perdas observadas após o processamento das dietas. A vitamina que se apresentou mais resistente ao processamento foi o ácido ascórbico e, durante a armazenagem, foi o $\alpha$-tocoferol.

\section{AGRADECIMENTOS}

Á FINEP, pelo auxílio financeiro para realização dos trabalhos científicos através da RECARCINE, bem como ao $\mathrm{CNPq}$, pela concessão das bolsas.

\section{REFERÊNCIAS BIBLIOGRÁFICAS}

AKIYAMA, D.M.; DOMINY, W.G.; LAWRENCE, A.L. Penaeid shrimp nutrition. In: . Marine shrimp culture: principles and practices. Amsterdam: Elsevier Science, 1992. p.535-568.

BARBIERI JÚNIOR, R.C.; OSTRENSKY NETO, A. Camarões marinhos: engorda. Viçosa, MG: Aprenda Fácil, 2002. 370p.

BISPO, D.L.N.; PEREIRA, O.C.M. Importância do conhecimento das alterações induzidas pelo estresse em animais domésticos. Interciencia, v.19, n.2, p.72-74, 1994.

BOYD. C.E. Composição da água e manejo do viveiro de camarão. Revista ABCC, Recife, v.3, n.1 p.17-19, 2001.

CONKLIN, D.E. Vitamins. In: . Crustacean nutrition advances in world aquaculture society. Lousiana: Baton Rouge, 1997. v.6, p.123-149.

FARIA, D.E.; JUNQUEIRA, O.M. Enfermidades nutricionais: doenças das aves. FACTA-Fundação Apinco de Ciência e Tecnologia Avícola, Campinas, v.1, p.431-448, 2000.

GADELHA, R.G.F.; PRADO, J.P.S.; CAVALHEIRO, J.M. $O$. Farinha de bagaço de cevada em dietas para engorda de camarões marinhos. Ciência Rural, Santa Maria, v.40, n. 1,2010

GARCIA, T.; PENTEADO, M.; CAMARGO, V. Qualidade de balas de gelatina fortificada com vitamina A, C e E. Ciência e Tecnologia de Alimentos, Campinas, v.25, n.4, p.743-749, out./dez. 2005.
GOMIS, D.B.; FERNÁNDEZ, M.P.; GUTIÉRRÉZ ALVAREZ, M.D. Simultaneous determination of fatsoluble vitamins and provitamins in Milk by microcolumn liquid chromatography. Journal of Chromatography A, Amsterdam, v.891, p.109-114, 2000.

GUILHERME, R.F.; CAVALHEIRO, J.M.O.; BRASILEIRO, O.L.; PRADO, J.P.S.; CAVALHEIRO, T.D. Composição e cinética de degradação do ácido ascórbico em rações para aquicultura. Ciência e Agrotecnologia, Lavras, v.33, n.4, 2009.

HE, H.; LAWRENCE, A.L.; LIU, R. Evaluation of dietary essentiality of fat-soluble vitamins, A, D, E and K for penaeid shrimp (Penaeus vannamei). Aquaculture, Amsterdam, v.103, p.177-185, 1992.

HUO, J.Z.; NELIS, H.J.; LAVENS, P.; SORGELOOS, P.; LEENHEER, A.P. Simultaneous determination of áTocopheryl Acetate and Tocopherols in aquatic organisms and fish feed. Journal of Chomatography B, Amsterdam, v.724, p.249-255, 1999.

LEE, M.H.; SHIAU, S.Y. Dietary vitamin C and its derivites affect immune responses in Grass shrimp, Penaeus monodon. Fish \& Shellfish Immunology, v.12, p.119-129, 2002.

LEITE, J.L.B.; RODRIGUES, P.B.; FIALHO, E.T.; FREITAS, R.T.F.; NAGATA, A.K.; CANTARELLI, V.S. Efeito da peletização e adição de enzimas e vitaminas sobre o desempenho e aproveitamento da energia e nutrientes em frangos de corte de 1 a 21 dias de idade. Ciência e Agrotecnologia, Lavras, v.32, n.4, p.1292-1298, jul./ago. 2008.

MARCHETTI, M.; TOSSANI, N.; MARCHETTI, S.; BAUCE, G. Stability of crystalline and coated vitamins during manufacture and storage of fish feeds. Aquaculture Nutrition, v.5, p.115-120, 1999.

NUNES, A.J.P. O cultivo de camarões marinhos no Nordeste do Brasil. Panorama da Aquicultura, Rio de Janeiro, v.3, p.26-33, 2001.

PEIL, S.Q.; POUEY, J.L.O.F.; LOPES, P.R.S.; MARTINS, C.R.; TIMM, G. Adição de vitamina A na dieta de póslarvas de Jundiá (Rhamdia quelen). Biodiversidade Pampeana, Uruguaiana, v.5, n.1, p.9-15, 2007. 
PRADO, J.P.S. Composição e estabilidade das vitaminas A e E em rações para camarão. 2008. Dissertação (Mestrado em Ciência e Tecnologia de Alimentos)-Universidade Federal da Paraíba, João Pessoa, 2008.

QIAN, H.; SHENG, M. Simultaneous determination of fat-soluble vitamins A, D and E and pro-vitamin D2 in animal feeds by one-step extraction and highperformance liquid chromatography analysis. Journal of Chromatography A, Amsterdam, v.825, p.127-133, 1998.

RANGANA, S. Manual of analysis of fruit and vegetable products. Tata: McGraw Hill, 1979.
RIBEIRO, E.P.; SERAVALLI, E.A.G. Química de alimentos. São Paulo: E. Blücher, 2004. 184p.

SACKHEIM, G.I.; LEHMAN, D.D. Química e bioquímica para ciências biomédicas. 8.ed. São Paulo: Manole, 2001. 644p.

SPSS. INC 11.0 for Windows: LEAD technologies. [S.1.], 2001. Software.

UNITES STATES PHARMACOPEIA. The national formulary: NF 23. Rockville, 2004. 2354p.

WALDIGE, V.A. A indústria de rações: a situação atual e as perspectivas. Panorama da Aquicultura, Rio de Janeiro, v.13, n.76, p.37 